\title{
Nonalcoholic fatty liver disease and alcoholic liver disease: metabolic diseases with systemic manifestations
}

\author{
Alexander J. Kovalic ${ }^{1}$, George Cholankeril ${ }^{2}$, Sanjaya K. Satapathy ${ }^{3}$ \\ ${ }^{1}$ Department of Internal Medicine, Wake Forest Baptist Medical Center, Winston Salem, North Carolina, USA; ${ }^{2}$ Division of Gastroenterology and \\ Hepatology, Stanford University School of Medicine, Stanford, CA, USA; ${ }^{3}$ Department of Internal Medicine, Division of Hepatology, Sandra Atlas \\ Bass Center for Liver Diseases \& Transplantation, Barbara and Zucker School of Medicine for Hofstra/Northwell Health, Manhasset, NY, USA \\ Contributions: (I) Conception and design: SK Satapathy; (II) Administrative support: None; (III) Provision of study materials or patients: None; \\ (IV) Collection and assembly of data: AJ Kovalic; (V) Data analysis and interpretation: All authors; (VI) Manuscript writing: All authors; (VII) Final \\ approval of manuscript: All authors. \\ Correspondence to: Sanjaya K. Satapathy, MBBS, MD, DM, MS(Epi), FACG, FASGE, AGAF, FAASLD. Sandra Atlas Bass Center for Liver Diseases \\ \& Transplantation, Barbara and Zucker School of Medicine for Hofstra/Northwell Health, 400 Community Drive, Manhasset, NY 11030, USA. \\ Email: ssatapat@northwell.edu.
}

\begin{abstract}
The progression of liver disease is portrayed by several common, overarching signs and symptoms. Classically, these include findings such as spider angiomata, jaundice, palmar erythema, and as cirrhosis decompensates, ascites, variceal hemorrhage ( $\mathrm{VH})$, hepatic encephalopathy (HE), and hepatocellular carcinoma (HCC). Aside from these universal hallmarks among cirrhotics, patients with nonalcoholic fatty liver disease (NAFLD) and alcoholic liver disease (ALD) harbor their own distinct systemic associations and manifestations. NAFLD is tightly linked to metabolic syndrome, which appears to be a driving force for a multitude of comorbidities, such as insulin resistance, cardiovascular disease, chronic kidney disease (CKD), obstructive sleep apnea (OSA), as well as increased malignancy risk. ALD also maintains a variety of comorbidities congruent with systemic effects of chronic alcohol use. These findings are highlighted by cardiovascular conditions, neuronal damage, myopathy, nutritional deficiencies, chronic pancreatitis, in addition to increased malignancy risk. While a general, guideline-driven management for all cirrhotic patients remains imperative for minimizing risk of complications, a tailored treatment strategy is useful for patients with NAFLD and ALD who entertain their own constellation of unique systemic manifestations.
\end{abstract}

Keywords: Nonalcoholic fatty liver disease (NAFLD); alcoholic liver disease (ALD); systemic

Received: 03 June 2019; Accepted: 13 August 2019; Published: 03 September 2019.

doi: $10.21037 / \operatorname{tgh} .2019 .08 .09$

View this article at: http://dx.doi.org/10.21037/tgh.2019.08.09

\section{Introduction}

The progression of liver disease and cirrhosis have a multitude of extrahepatic manifestations. These findings include spider angiomata, Terry's nails, palmar erythema, hepatosplenomegaly, jaundice, scleral icterus, and caput medusa (see Table 1) $(1,2)$. As patients with this disease continue to deteriorate, signs of decompensation arise as defined by the presence of ascites, spontaneous bacterial peritonitis (SBP), variceal hemorrhage $(\mathrm{VH})$, and hepatic encephalopathy (HE). These clinical clues materialize along a similar trajectory towards a common endpoint of end stage liver disease, which also harbors increased risk for hepatocellular carcinoma (HCC) (3).

Both nonalcoholic fatty liver disease (NAFLD) and alcoholic liver disease (ALD) represent two common etiologies of liver disease and cirrhosis. Although both conditions ultimately share common endpoints of decompensated and end stage liver disease as addressed 
Table 1 Common systemic findings ubiquitously seen among progression of cirrhosis of all etiologies

Constitutional symptoms
Fatigue/weakness
Obesity
Anorexia
Weight loss
Sarcopenia
Neurologic
Asterixis
HE
Cardiovascular

Decreased effective intra-arterial pressure

Cirrhotic cardiomyopathy

Peripheral edema

Clubbing and hypertrophic osteoarthropathy

Sequela of portal hypertension

Hepatosplenomegaly

Ascites

Caput medusa

Esophagogastric varices

Internal hemorrhoids

Mesenteric vein thrombosis

Head and neck

Fetor hepaticus

Dermatologic

Jaundice and/or scleral icterus

Spider angiomata

Telangiectasia

Palmar erythema

Terry's nails

Muehrcke's nails

Decreased body hair

Serologic testing

Hyponatremia

Hypoalbuminemia

Elevation of liver chemistries (AST, ALT, alkaline phosphatase, bilirubin)

Table 1 (continued)
Table 1 (continued)

Hematologic

Deficiency of procoagulant (protein C \& S) and anticoagulant factors (factors II, V, VII, VIII, IX, X, XII)

Thrombocytopenia

Anemia

Leukopenia

Hyperglobulinemia

Reproductive/genitourinary

Testicular atrophy

Gynecomastia

Increased malignancy risk

HCC

HE, hepatic encephalopathy; HCC, hepatocellular carcinoma; AST, aspartate aminotransferase; ALT, alanine aminotransferase.

above, NAFLD and ALD accommodate unique features both in their presenting diagnoses and as well as throughout the development of the disease course. While initially triggered by metabolic disturbances, several distinct systemic manifestations of both NAFLD and ALD have become hallmarks of the diagnosis and management of these conditions.

\section{NAFLD}

\section{Pathogenesis and association with metabolic syndrome}

The interwoven affinity of NAFLD with metabolic syndrome has firmly been established. Peripheral insulin resistance, obesity, dyslipidemia, and altered lipid metabolism synergistically catalyze the accumulation of hepatic triglycerides, which ultimately generates the lipotoxicity and insistent inflammation necessary for the progression towards nonalcoholic steatohepatitis (NASH) and cirrhosis.

One large meta-analysis determined among a pooled population of 81,411 patients across eight studies that patients diagnosed with NAFLD harbored a significantly elevated risk for development of metabolic syndrome within a median 4.5 year follow up (4). This relative risk was calculated to be 1.80 (95\% CI: 1.89-2.07) based upon alanine aminotransferase levels, 1.98 (95\% CI: 1.89-2.07) based upon GGT, and 3.22 (95\% CI: 3.05-3.41) when diagnosed via ultrasonography. Another recent systematic 
review and meta-analysis established that obesity portends worse prognosis among NAFLD patients, however no direct correlation was observed among degree of obesity with severity of NAFLD (4).

Perhaps the most potent association, however, is that of type 2 diabetes mellitus (T2DM) with NAFLD. By itself, T2DM carries its own slew of comorbidities and elevated cardiovascular risk. One recent meta-analysis calculated a HR 2.22 (95\% CI: 1.84-2.60) with respect to NALFD patients' risk of developing T2DM (5). Hepatic steatosis and ensuing lipotoxicity produce a state of hyperglycemia, dyslipidemia, and a proinflammatory environment that catalyzes the development of insulin resistance, atherosclerosis, and pancreatic beta-cell dysfunction and apoptosis (6). One recent meta-analysis from China, revealed an accelerated increase in NAFLD over a 10 -year period, possibly attributing this rise to increased prevalence of metabolic syndrome and T2DM (7).

Overall, there is an intimate connection of NAFLD and metabolic syndrome, with particular respect to T2DM, which catalyzes the continuous insult of hepatic steatosis, lipotoxicity, fibrosis, and progression towards NASH.

\section{Adipokines}

In addition to the presence of metabolic syndrome and obesity, the increasing role of adipokines has specifically been shown to fuel this pathogenesis and other findings seen in NAFLD (8). The mere presence of triglycerides and release of free fatty acids contributes to dyslipidemia. However, the endocrine-mediated release of other hormones from adipose tissue have been shown to be increased, such as resistin, RBP4 (retinol-binding protein 4), leptin, and 11 HSD-1, in addition to a decrease in adiponectin, which all perpetuate the function of insulin resistance (9).

Furthermore, elevated release of the adipokines PAI-1 (10) and angiotensinogen (11) place NAFLD patients at an increased propensity for thrombosis and atherosclerosis, respectively. Adipokines also fuel a proinflammatory state via the secretion of IL-6, TNF- $\alpha$, and CCL2, which not only stimulates a local injury and fibrotic response, but also a number of systemic conditions $(12,13)$.

The multi-faceted inputs from adipokines clearly augments NAFLD pathogenesis in addition to the development of common systemic manifestations seen in this disease.

\section{Cardiovascular disease}

A bevy of preliminary data had originally suggested this association, however more robust evidence has firmly planted the rooting of atherosclerosis with NAFLD. Two original systematic reviews were able to confirm these findings based upon increased carotid intima media thickness, endothelial dysfunction, arterial stiffness, and coronary calcification among NAFLD subjects $(14,15)$. Similar findings among subsequent systematic reviews/ meta-analyses were replicated across a wide range of patient populations (16-22).

There has been an overall trend towards increased risk of $\mathrm{CV}$ events among NAFLD patients, such as acute coronary syndrome (ACS), cerebrovascular accident (CVA), or peripheral artery disease (PAD), however there were a minor discordance of the statistical significance of these findings among individual observational studies (23-27). Notwithstanding these differences, more robust evidence has been performed across three systematic review/metaanalyses that have settled this discussion. One Chinese meta-analysis reported an increased risk of prevalent (OR 1.81, 95\% CI: 1.23-2.66) and incident (HR 1.37, 95\% CI: $1.10-1.72) \mathrm{CV}$ events, however no overall difference in mortality (19). Another Italian meta-analysis revealed an increased risk of CV events with OR 1.64 (95\% CI: $1.26-$ 2.13) (28). The risk of $\mathrm{CV}$ events appeared to increase with the severity of NAFLD with OR 2.58 (95\% CI: 1.78-3.75) based upon imaging and histologic parameters. A third systematic review and meta-analysis found an elevated risk of CV events (RR 1.77, 95\% CI: 1.26-2.48) and CV mortality (RR 1.46, 95\% CI: 1.31-1.64) among NAFLD patients as compared to controls. While most of this data was performed across observational studies where causality cannot be surmised, the risk of CV events among NAFLD patients seems to be verified across multiple, large-scale systematic reviews and meta-analyses.

Meanwhile, further data illuminate the role of T2DM in this process by analyzing the synergistic increase in $\mathrm{CV}$ risk in the presence of both NAFLD and T2DM (29). This concept introduces a limitation we called attention to in a prior review (30). That is, it is difficult to delineate the $\mathrm{CV}$ risk specifically from NAFLD apart from its comorbidities based upon current studies, in particular, metabolic syndrome, obesity, insulin resistance, and T2DM. There appears to be a large discrepancy on the prevalence of T2DM between NAFLD and control subjects in a number of these studies. And even among studies exclusively 
performed in nondiabetic patients, there is a great disparity of insulin resistance observed between NAFLD and control patients. Overall, there is an increase in $\mathrm{CV}$ diseases among NAFLD patients, however the pathogenesis and causality of these disease processes remain in question given the substantial confounding influence of metabolic syndrome and T2DM observed in these studies.

\section{Venous thromboembolism}

Cirrhotic coagulopathy is a complex topic which actually portends increased overall risk towards both bleeding and thrombosis owning to the loss of procoagulants and anticoagulants, respectively. However, NAFLD provides a unique perspective further complicating this coagulopathic snafu. Multiple studies have identified the presence of NAFLD as a risk factor for thrombosis and unprovoked veno-thromboembolism (VTE) (31-33). While there are currently no prospective or randomized trials to date, one Italian, cross sectional study was able to establish interesting findings towards coagulopathy and propensity towards procoagulation (34). A total of 113 patients, who had previously had liver biopsy, underwent further testing of thrombin, coagulation factors, and protein $\mathrm{C}$ activity. One of the markers for procoagulation and thrombosis formation was defined by the endogenous-thrombinpotential (ETP), which was statistically increased along the NAFLD spectrum among control subjects, hepatic steatosis, and cirrhosis with values of $0.57,0.72$, and 0.80 , respectively $(\mathrm{P}<0.001)$. Among this cohort, there was no significant difference in ETP of patients with NASH cirrhosis as compared to other etiologies of cirrhosis, such as alcoholic or viral. Perhaps the presence of cirrhosis or obesity represent confounding factors in this study, however it appears that there is increased predilection for VTE as the severity of NALFD progresses.

\section{Chronic kidney disease (CKD)}

Many cohort studies have shown an association between CKD and NAFLD. This may serve as an unsurprising finding given the large amount of concomitant metabolic syndrome, obesity, diabetes mellitus, and atherosclerosis in this patient population. Perhaps the most robust data with respect to this topic lies within two systematic reviews/ meta-analyses.

In the first by Musso et al. 2014, it was demonstrated that patients with NAFLD had both an elevated prevalent
(OR 2.12, 95\% CI: 1.69-2.66) and incident (HR 1.79, 95\% CI: 1.65-1.95) CKD risk (35). The presence of NASH was found to additionally increase the prevalence of CKD (OR 2.53, 95\% CI: 1.58-4.05). No statistically significant heterogeneity was present in this analysis. However, major differences in the number of biopsy-proven NAFLD patients, possibility of selection bias, and utilization of ultrasound and/or liver chemistries for the selection of controls among some studies may have inappropriately misclassified patients and limited the external validity of this meta-analysis.

In the only other meta-analysis to date, Mantovani et al. 2018 also illustrated the close link of NAFLD and CKD (36). Patients with NAFLD were found to have HR 1.37 (95\% CI: 1.20-1.53) for CKD development as compared to controls. This risk appears to increase with worsening NAFLD steatosis and fibrosis. However, it is important to note that most of the studies in both of these meta-analyses were observational, and thus, the strong association of NAFLD and CKD development should not be mistaken for causality of these two disease processes.

\section{Polycystic ovarian syndrome (PCOS)}

There has been mounting evidence in the recent literature anchoring the association of PCOS with NAFLD. A multitude of studies among recent years have characterized this association, which has been further validated through three major systematic reviews and/or meta-analyses. The first study was a systematic review in 2011 that reported the current literature on the topic (37). The interplay among PCOS, NAFLD, insulin resistance, hyperandrogenism, and obesity was clearly present. However, it was noted that most studies observed a proportionate rise in prevalence of PCOS and NAFLD with the degree of insulin resistance and adipose tissue. A second systematic review and metaanalysis performed across 17 studies and included 2,700 PCOS patients with matched controls (38). PCOS patients were found to have higher prevalence of NAFLD as compared to controls with OR 2.54 (95\% CI: 2.19-2.95). These studies revealed an overall association with elevated serum androgen levels, obesity, and insulin resistance among patients with both PCOS and NAFLD. Finally, another meta-analysis solidified this association, citing an OR 2.25 (95\% CI: 1.95-2.60) among patients with PCOS and the risk of NAFLD (39). This meta-analysis also found an increased association of PCOS patients with hyperandrogenism with development of NAFLD. 
Interestingly, PCOS patients without hyperandrogenism were not found to have increased risk of NAFLD (OR 1.46, 95\% CI: 0.55-3.87), suggesting elevated serum androgen levels are integral in this pathogenesis. Overall, current literature demonstrates a robust relationship between the presence of NAFLD and PCOS, and this link appears to be heightened in the setting of hyperandrogenism, obesity, and insulin resistance.

\section{Obstructive sleep apnea (OSA)}

Undoubtedly intertwined to its close connection to metabolic syndrome, NAFLD patients have been demonstrated to have increased frequency of OSA. This association has been validated through its first systematic review and meta-analysis including 2,183 patients, which revealed increased risk of OSA not only among the presence, but also the severity of NAFLD (40). These included increased OSA risk among NAFLD patients with OR 2.01 (95\% CI: 1.36-2.97), 2.99 (95\% CI: 1.79-4.99), 2.36 (95\% CI: $1.46-3.82$ ), and 2.60 (95\% CI: $1.88-3.61$ ) when diagnosed by histology, imaging, AST, and ALT elevation, respectively. Another recent systematic review and meta-analysis illustrated that OSA was tightly linked to degree of hepatic steatosis, lobular inflammation, ballooning degeneration, and fibrosis among biopsy-proven NAFLD patients (41). This evidence not only suggests that OSA is a common manifestation seen among NAFLD patients, but also that this association proportionately strengthens along the continuum of NAFLD severity.

\section{Osteopenia}

Sedentary lifestyle more commonly observed among NAFLD patients (42) may lead to increased osteoporosis (43). However, several other aberrancies in bone metabolism among NAFLD subjects have been noted.

Osteocalcin is a protein produced by osteoblasts in order to promote bone matrix formation (44). Osteocalcin has been shown to be decreased in a severity-dependent relationship with NAFLD $(45,46)$. Osteoprotegerin is a glycoprotein that inhibits osteoclast activity and promotes osteoclast apoptosis via a RANKL (receptor activator of nuclear factor kappa-B ligand) mediated process (47). It has been established that osteoprotegerin is decreased in NAFLD (48). Osteopontin modulates osteoblast and osteoclast activity in order to inhibit mineral crystal growth, enhance bone resorption, and decreased bone deposition (49). Osteopontin has been found to be increased among NAFLD subjects (50).

Overall, the presence of sedentary lifestyle and aberrancies in multiple bone hormones construct an ideal foundation for osteoporosis among NAFLD patients.

However, these findings have not been corroborated in future studies on this topic. In a study performed by Upala et al. (51), there was no significant difference calculated between NAFLD and bone mineral density as compared to controls. Body mass index (BMI) was found to be the variable that most strongly correlated with variations in bone mineral density.

Another large systematic review and meta-analysis revealed an increase in osteoporotic fractures among NAFLD patients, however this was not associated with low bone mineral density itself (52).

While several confounding factors exist among this population with a high prevalence of obesity and sedentary lifestyle, more studies isolating the differences between bone mineral density, osteoporosis, and NAFLD are required to further delineate this relationship.

\section{Nutritional considerations}

NAFLD has not had a classical association with nutritional deficiencies per se, however have been more linked with obesity, diabetes, and excessive caloric intake.

One large systematic review and meta-analysis revealed that hypercaloric fructose and glucose diets were found to increase hepatic triglyceride content and raise liver chemistries (53). However, these diets appear to be confounded by overall excessive caloric intake, more so than by the intake of sugars themselves, which potentially skews the external validity of this study. Another large systematic review and meta-analysis conducted in 1,361 NAFLD cases with matched controls unveiled a strong link of NAFLD with sugar sweetened beverage consumption (54). Finally, another systematic review and meta-analysis illustrated that while no reduction in liver chemistries were observed among patients with NAFLD, a low carbohydrate diet decreased intrahepatic lipid content by $11.53 \%$ (95\% CI: -18.10, -4.96) (55). Furthermore, a decrease in copper bioavailability has been demonstrated to contribute to atherosclerosis among NAFLD patients (56).

Overall, there is fairly robust evidence to validate the association of excess dietary sugar and carbohydrate intake on NAFLD development, which is particularly compounded in the presence of metabolic syndrome, obesity, and T2DM. 


\section{Increased malignancy risk}

Aside from the overall increased incidence of HCC observed with progression of cirrhosis, two major systematic reviews and meta-analyses have shed light upon increased malignancy risk observed among NAFLD patients. One study analyzed seven case-control studies comparing a total of 9,102 patients with cholangiocarcinoma against 129,111 controls (57). NAFLD was identified as a risk factor for the development of both intrahepatic (OR 2.22, 95\% CI: 1.52-3.24) and extrahepatic cholangiocarcinoma (OR 1.55, 95\% CI: 1.03-2.33), respectively. However, the slightly increased predilection towards intrahepatic cholangiocarcinoma may suggests a similar pathogenic mechanism with HCC. Another major analysis performed across 11 observational studies concluded that NAFLD was associated with a significantly increased risk of prevalent and incident colorectal adenomas and colorectal cancer (58). This increased risk was found to be independent of age, gender, smoking history, BMI, diabetes, or metabolic syndrome. These two major systematic reviews and metaanalyses illuminate the association of NAFLD and increased malignancy risk, however, these analyses were performed primarily across observational studies from which causality cannot be inferred. Additionally, there has also been a documented association of NAFLD with HCC, however this risk is particularly more prevalent among patients with cirrhosis (59). There appears to be an overall link between obesity and cancer, perhaps through the underlying proinflammatory presence $(60,61)$. However while revisiting the mounting evidence of adipokines, a recent study has demonstrated a strong link between adiponectin and leptin and the development of obesity-related cancers (62). Although this remains a topic still in the dawn of formation, there persists a strong impetus for malignancy among NAFLD patients from a variety of perspectives.

\section{Other}

NAFLD patients have also been found to have an increased predilection for the development of other medical conditions, including psoriasis, irritable bowel syndrome (IBS), asthma, and depression. Interestingly, one study published increased hepatic fibrosis among patients with psoriasis versus NAFLD patients who did not have psoriasis (63). This mechanism still remains relatively unknown. However, this association was also validated in a meta-analysis where patients with psoriasis had an increased risk of developing NAFLD as compared to non-psoriatic controls (OR 2.15, 95\% CI: 1.57-2.94) (64). The link between IBS (65) and asthma (66) among NAFLD patients has also been suggested, however there in an overall lack of large, well-powered data to support this link across all patient populations. Alternative data also proposes an increased incidence of depression among NAFLD patients, particularly as compared to alternative forms of liver disease such as hepatitis B (67).

\section{ALD}

\section{Pathogenesis}

The effect of ethanol-medicated hepatocellular damage is relatively unambiguous. This direct cellular injury lays the foundation for interminable inflammation, cell death, and fibrosis that ultimately results in cirrhosis and decompensated ALD. However, these effects synergize with alcohol-induced gut barrier dysfunction and ensuing elevation in gut-derived bacterial translocation into the portal circulation (68). In particular, the bacterial endotoxin lipopolysaccharide (LPS) goes on to activate Toll-like receptors (TLR) on hepatocytes and Kupffer cells in order to generate a vigorous release of proinflammatory cytokines $(69,70)$. In tandem with the direct toxicity of ethanol itself, it is this production of proinflammatory mediators, formation of reactive oxidative species (ROS), and subsequent cellular injury that not only progress ALD pathogenesis, but also incite many of the systemic manifestations so closely tied to this disease process (71).

Within the hepatocyte, ethanol is primarily metabolized to acetaldehyde via alcohol dehydrogenase (ADH). However, when this pathway is overwhelmed, increasingly more amounts of ethanol are digested by the microsomal ethanol oxidizing system (MEOS) in the endoplasmic reticulum (72). Utilization of the MEOS has an increased propensity for the formation of not only acetaldehyde, but also other toxic byproducts, such as ROS and protein adducts. Once ethanol is metabolized, acetaldehyde can then go on to be catalyzed by acetaldehyde dehydrogenase (ALDH), thus forming acetate. However, the amount and chronicity of ethanol ingested dictate the overall kinetics and enzymes employed, which directly correlate to the amount of ethanol, acetaldehyde, ROS, and protein adducts that mediate the primary toxicity emanating from this pathway. 


\section{Acid-base disorders}

Ingestion of alcohol has the ability to create a state of alcoholic ketoacidosis (73). In addition to ketoacidosis, three other primary mechanisms seen in advancing ALD: hypoalbuminemia which precipitates a metabolic alkalosis, an aberrant Cori cycle which causes a buildup of lactic acid, and hyperventilation (74). Hyperventilation can be from a number of driving forces, such as central activation of estrogen receptors, HE, intrapulmonary pathology, or simply from diaphragm elevation in the setting of ascites. All of these factors influencing an alkalotic or acidotic milieu are generally kept in balance among stable cirrhotic patients. However, insults such as critical illness or decompensating liver failure can alter this balance creating unique acid-base disturbances depending on the inciting pathology.

\section{Peripheral neuropathies and CNS pathology}

Chronic effects of alcohol use can lead to a multitude of systemic effects. There is a direct effect of ethanol and its byproducts, which alters lipid function, generates ROS, increased mitochondrial damage, and ultimately leads to neuronal cell injury or death (75). Furthermore, ethanol is able to alter intracellular neuronal communications, which normally foster cell growth and differentiation. These direct effects of ethanol commonly manifest as cerebellar ataxia and alcoholic neuropathy, either in the central nervous system including brain atrophy (76), or peripheral neuropathy. Peripheral neuropathy typical presents in a classic "stocking-glove" pattern with early signs of paresthesias. As disease progresses under the influence of chronic alcohol use, proprioceptive impairments occur after deep tendon reflexes are lost, which ultimately may manifest as muscle weakness and atrophy once motor fibers are damaged (77). This axonal neuron damage oftentimes occurs in tandem with nutritional deficiencies, commonly folate and vitamin B12 (78). Similarly, nutritional deficiencies can also precipitate CNS findings, most notably from thiamine deficiency as seen in WernickeKorsakoff encephalopathy. This condition typically presents acutely with gait ataxia, cognitive changes, with or without nystagmus, and even coma in severe circumstances (75). These findings generally improve after administration of supplemental thiamine. Lastly, the progression of worsening liver disease due to chronic alcohol use often leads to a buildup of ammonia and manganese, which can promote neurotoxicity due to increased oxidative stress and changes in cerebral blood flow (79). Overall, the consequences of liver disease due to chronic alcohol abuse can lead to central and peripheral neuropathies secondary to both direct toxic effects and vitamin deficiencies.

\section{Cardiovascular disease}

Patients with ALD invariably develop concomitant cardiac conditions. The link between alcohol and hypertension has been well documented. A recent systematic review and meta-analysis has shown that there is a dose-dependent relationship with alcohol reduction on blood pressure improvement (80).

Alcohol also has a profound association with the development of cardiac arrhythmias, most notably atrial fibrillation. Four major systematic reviews and metaanalyses have been performed highlighting this link. In the first study, it was shown that alcohol exerted a dosedependent relationship that elevated risk of atrial fibrillation with increasing doses of alcohol (81). A second metaanalysis corroborated these dose-dependent effects of alcohol on atrial fibrillation (82). A third study has reported a OR of 1.51 (95\% CI: 1.31-1.74) among alcohol use and the association with atrial fibrillation (83). Lastly, a recent systematic review and meta-analysis stratified alcohol use to low (up to one standard drink per day), moderate (1-2 standard drinks per day), and high. This analysis demonstrated no association with atrial fibrillation in the low alcohol intake group with HR 0.95 (95\% CI: 0.85-1.06), elevated risk of atrial fibrillation in the male, moderate alcohol intake group with HR 1.26 (95\% CI: $1.04-1.54$ ) but not the females, and finally an overall association of high alcohol intake and incident atrial fibrillation with HR 1.34 (95\% CI: $1.20-1.49)$ (84). Clearly, there appears to be a direct relationship to the amount of alcohol ingested and the link to atrial fibrillation.

While some conflicting data exist regarding potential beneficial effects of light-to-moderate alcohol consumption, another cardiac manifestation commonly seen among patients with ALD is dilated cardiomyopathy. These cardiac findings generally present as a dilated left ventricle, increased left ventricular mass, with normal or reduced systolic dysfunction (85). These cardiac manifestations are not to be confused with cirrhotic cardiomyopathy, as seen with advanced cirrhosis of any etiology, which is typically characterized by impaired relaxation, diastolic dysfunction, 
and hyperdynamic cardiac changes resulting in increased cardiac output (86). Heavy chronic alcohol intake continues to have a strong association with cardiomyopathy and development of cardiac dysfunction $(87,88)$.

\section{Pulmonary abnormalities}

Given the side effects of alcohol use and altered sensorium, it may be unsurprising that patients with ALD are at risk for aspiration of gastric contents (89). This can lead to a multitude of other complications including pneumonia, empyema, and lung abscesses, traditionally stemming from pathogens endogenous to gastric or oropharyngeal flora (90). Furthermore, chronic alcohol abuse is a risk factor for transfusion-related acute lung injury (TRALI) and acute respiratory distress syndrome (ARDS) (91).

\section{Skeletal myopathies}

Patients with ALD can harbor several forms of myopathy throughout their disease course. While sarcopenia is a common manifestation as ALD progresses to advanced stages, this will be discussed below with the nutritional deficiencies and malabsorption. Alcohol appears to be a risk factor for the development of acute rhabdomyolysis (92). And even though this patient population is at increased risk for falls, the development of rhabdomyolysis has been shown to occur in a non-traumatic setting (93). The management of rhabdomyolysis poses some challenges among patients with ALD, particularly with respect to intravenous fluid resuscitation, volume status, and renal function. More chronic manifestations among ALD patients can be seen with alcoholic myopathy. Chronic alcohol intake fuels stunting of skeletal muscle formation and the promotion of autophagy through the effects of alcohol and its acetaldehyde byproducts $(94,95)$. Newer evidence also suggests a role of aberrant regenerative capacity of muscle progenitor cells contributing to alcohol-related skeletal muscle dysfunction (96).

\section{Nutritional deficiencies, malabsorption, and sarcopenia}

Patients with ALD oftentimes receive a majority of their caloric intake through the carbohydrates in alcohol, which is devoid of essential proteins, fats, and other micronutrients $(97,98)$. Heavy chronic alcohol use intrinsically can cause diarrhea, malabsorption, and chronic pancreatitis, further hindering nutrient reabsorption through limited initial intake (99). Certain vitamin deficiencies, as seen with vitamin A and zinc, can alter taste and smell (100), and aberrant levels of glucose, leptin, and ghrelin can affect overall satiety (101). Both alcohol and acetaldehyde also stunt skeletal muscle formation and promote autophagy $(94,95)$. Not only does cirrhosis constitute a proinflammatory state, but it is also characterized by a hypermetabolic state (102). Furthermore, as liver disease advances, hyperammonemia also induces sarcopenia via a mTORC1-regulated pathway (103). Overall, there are several mechanisms that converge and contribute to decreased caloric intake, nutritional deficiencies, weight loss, and sarcopenia often seen among this patient population (104).

Specifically, there are a number of nutrient and vitamin deficiencies classically found among patients with ALD (100). Vitamin A deficiency has the ability to lead to night blindness and increased fibrosis. Thiamine deficiency contributes to the aforementioned Wernicke-Korsakoff syndrome, in addition to neuropathy and potentially heart failure. Diminishing levels of vitamin B3 give rise to pellagra, a syndrome commonly characterized by dermatitis, diarrhea, and dementia. Vitamin B6 deficiency fosters symptoms that include a rash, glossitis, neuropathy, and sideroblastic anemia. Vitamin B9 deficiency can lead to macrocytic anemia. Low levels of vitamin B12 similarly can present as macrocytic anemia, in addition to other findings, such as neuropathy and weakness. Loss of adequate vitamin C levels traditionally present with scurvy. Vitamin D deficiency manifests as decreased bone mineral density and osteoporosis. Vitamin E insufficiency typically engenders immune cell dysfunction, myopathy, and neurologic defects. Zinc deficiency harbors changes consistent with skin lesions, altered taste, change in smell, encephalopathy, hypogonadism, alterations in immune function, and impaired wound healing (105).

\section{Chronic pancreatitis and pancreatic insufficiency}

The impetus of chronic alcohol intake promotes the risk of acute pancreatitis, which overtime can manifest as chronic pancreatitis after repeated episodes. The continual exposure of alcohol and steady foundation of inflammation leads to "burn out" of these pancreatic cells that ultimately harbors both exocrine and endocrine pancreatic insufficiency. Exocrine pancreatic insufficiency is characterized by malabsorptive diarrhea that also contributes to decreased vitamin intake (106). The progression of exocrine 
insufficiency is heightened with concomitant tobacco abuse, and thus, the first line in management is unsurprisingly cessation of alcohol and tobacco products (107). The potential simultaneous involvement and loss of pancreatic islet cells engenders endocrine insufficiency and progression towards diabetes mellitus. However, this modality of diabetes poses a unique quandary in the sense that there is a coexisting loss of glucagon secretion, which further portends episodes of hypoglycemia (108). In the setting of continued alcohol intake, this can be a difficult facet of ALD to manage given the elevated carbohydrate content among alcoholic beverages, particularly beer. Overall, the caveats of exocrine and endocrine pancreatic insufficiency portend a difficult situation in the management of ALD.

\section{Osteopenia}

Although it appears patients with cirrhosis are at increased risk for altered bone mineral density and microarchitecture, regardless of etiology (109), patients with ALD harbor a multitude of unique risk factors for the development of osteopenia. As previously mentioned, hypoalbuminemia and nutritional deficiencies construct an ideal milieu for altered bone health and turnover. In particular, vitamin $\mathrm{D}$ and zinc deficiencies appear to be integral to this process (110). Malabsorption in the setting of chronic pancreatitis further exacerbates this mechanism, with a recent study also highlighting a potential association of vitamin $\mathrm{D}$ and $\mathrm{K}$ deficiencies with osteoporosis (111). Alcohol-induced myopathy and neuropathy provide a lack of normal mechanical stressors on bone, leading to alterations in bone architecture and density (112). Lastly, alcohol abuse also portends increased risk of falls and trauma ultimately resulting in increased bone fractures among this patient population.

Ethanol also provides direct toxic effects to primary cellular pathways of bone formation and resorption. Both ethanol and acetaldehyde have been demonstrated to provide oxidative damage leading to reduced osteoblast function and bone formation (113). Simultaneously, through both an auspicious milieu of proinflammatory cytokines and also the stimulation of the RANK (receptor activator of NF-kB) pathway, there is an overall activation of osteoclast activity seen among patients with ALD (114). The synergistic effects upon osteoblasts and osteoclasts leading to decreased bone formation and increased bone resorption are responsible for the increased risk of osteopenia among this patient population. Yet, larger clinical studies have not yet captured this overall risk. In the only meta-analysis to date regarding ALD and bone health, patients with ALD were found to have an elevated rate of bone fractures with RR 1.944 (95\% CI: 1.354-2.791) as compared to controls (115). And while there was a trend towards lower bone mineral density among ALD patients, there was no statistical difference found in the development of osteoporosis or bone mineral density as compared to controls. Nevertheless, it appears that these changes of osteopenia are reversed with abstinence from alcohol, which remains the first line treatment in the management of alcohol-related bone disorders (116).

\section{Increased malignancy risk}

In addition to HCC risk associated with cirrhosis, patients with ALD have been linked to many other forms of malignancy based upon persistent alcohol use. One large systematic review and meta-analysis demonstrated patients with chronic alcohol intake for at a two-fold increase for the general development of "cancer", however the subtypes were not stratified (117). Further evidence has established a dose-dependent alcohol intake with oropharyngeal, laryngeal, esophageal, colorectal, pancreatic, and breast cancer (118-120). This elevated malignancy risk not only is imperative to screen and treat patients with ALD appropriately, but can also represent a challenging obstacle for patients ultimately requiring liver transplantation.

\section{Parotid gland hypertrophy and Dupuytren's contractures}

Two final systemic findings seen in a large proportion of patients with ALD include enlarged parotid glands and Dupuytren's contractures. Chronic alcohol appears to contribute to the hypertrophy of parotid glandular tissue. This hypertrophy is increased as compared to controls and diabetic subjects (121). Meanwhile, Dupuytren's contractures are areas of increased connective tissue and collagen deposition resulting in fibrosis typically characterized along the fourth and fifth digits of the palmar fascia (122). Dupuytren's contractures are tightly linked to chronic alcohol use and are not infrequently observed among patients with ALD (123).

\section{Conclusions}

In addition to common endpoints seen across all etiologies of cirrhosis, patients with NAFLD and ALD foster unique 
Table 2 Systemic manifestations specifically linked to NAFLD and ALD

\begin{tabular}{|c|}
\hline NAFLD \\
\hline Metabolic syndrome \\
\hline Obesity \\
\hline Insulin resistance \\
\hline T2DM \\
\hline Dyslipidemia \\
\hline Cardiovascular disease \\
\hline Hypertension \\
\hline Atherosclerosis \\
\hline Increased ACS, CVA, PAD \\
\hline Venous thromboembolism \\
\hline DVT \\
\hline PE \\
\hline CKD \\
\hline PCOS \\
\hline Hyperandrogenism \\
\hline OSA \\
\hline Osteoporosis \\
\hline Nutritional considerations \\
\hline Excessive dietary sugar and carbohydrate intake \\
\hline Overall increase daily caloric intake \\
\hline Increased malignancy risk \\
\hline Cholangiocarcinoma \\
\hline Colorectal carcinoma \\
\hline ALD \\
\hline Acid-base disorders \\
\hline Alcoholic ketoacidosis \\
\hline Hypoalbuminemia-induced metabolic alkalosis \\
\hline Increased lactic acid accumulation \\
\hline Hyperventilation-mediated respiratory alkalosis \\
\hline
\end{tabular}

Table 2 (continued)

systemic manifestations associated with their individual disease processes (see Table 2). The major catalyzing factor appears to be a close partnership with metabolic syndrome and the systemic effects of alcohol use, respectively. There are a bevy of metabolic disturbances, comorbidities,
Table 2 (continued)

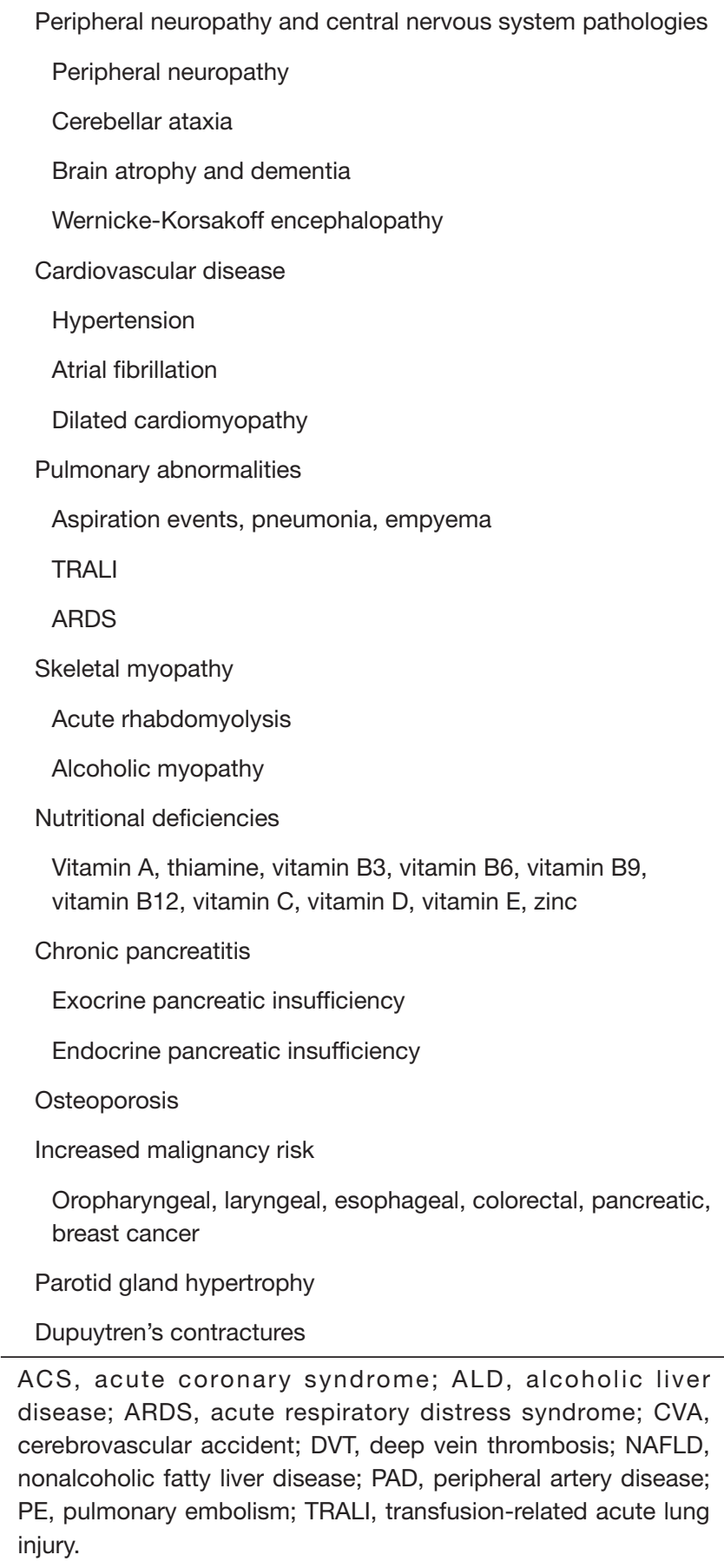

ACS, acute coronary syndrome; ALD, alcoholic liver disease; ARDS, acute respiratory distress syndrome; CVA, cerebrovascular accident; DVT, deep vein thrombosis; NAFLD, nonalcoholic fatty liver disease; PAD, peripheral artery disease; $\mathrm{PE}$, pulmonary embolism; TRALI, transfusion-related acute lung injury.

concomitant nutritional aberrancies, and increased malignancy risks exclusively associated with both NAFLD and ALD. A broader understanding of these two disease processes, which constitute some of the most common etiologies of cirrhosis, will ideally guide clinicians to 
focus on these unique systemic manifestations in order to promote a more comprehensive, and ultimately effective, treatment strategy for patients with NAFLD and ALD.

\section{Acknowledgments}

None.

\section{Footnote}

Conflicts of Interest: Dr. Satapathy reports receiving grants from Gilead Sciences and participated in Speakers Bureau, Advisory Board of Gilead Sciences, received grants from Intercept Pharmaceuticals and participated in the Speakers Bureau, Advisory Board of Intercept Pharmaceuticals, received grants from Shire, grants from Conatus, grants from Bayer, and participated in the Speakers Bureau of Alexion, received grants from Dova and participated in the Speakers Bureau, and Advisory Board of Dova, received grants from Genfit, grants from Exact Sciences, and participated in the Advisory Board of Abbvie, outside the submitted work. The other authors have no conflicts of interest to declare.

Ethical Statement: The authors are accountable for all aspects of the work in ensuring that questions related to the accuracy or integrity of any part of the work are appropriately investigated and resolved.

\section{References}

1. Ghany MG, Hoofnagle JH. Approach to the Patient with Liver Disease. In: Jameson JL, Fauci AS, Kasper DL et al. (eds). Harrison's Principles of Internal Medicine, 20e. New York, NY: McGraw-Hill Education, 2018.

2. Udell JA, Wang CS, Tinmouth J, et al. Does this patient with liver disease have cirrhosis? Jama 2012;307:832-42.

3. Marrero JA, Kulik LM, Sirlin CB, et al. Diagnosis, Staging, and Management of Hepatocellular Carcinoma: 2018 Practice Guidance by the American Association for the Study of Liver Diseases. Hepatology 2018;68:723-50.

4. Ballestri S, Zona S, Targher G, et al. Nonalcoholic fatty liver disease is associated with an almost twofold increased risk of incident type 2 diabetes and metabolic syndrome. Evidence from a systematic review and meta-analysis. J Gastroenterol Hepatol 2016;31:936-44.

5. Mantovani A, Byrne CD, Bonora E, et al. Nonalcoholic Fatty Liver Disease and Risk of Incident Type 2 Diabetes:
A Meta-analysis. Diabetes Care 2018;41:372-82.

6. Stefan N, Kantartzis K, Haring HU. Causes and metabolic consequences of Fatty liver. Endocr Rev 2008;29:939-60.

7. Zhou F, Zhou J, Wang W, et al. Unexpected Rapid Increase in the Burden of Nonalcoholic Fatty Liver Disease in China From 2008 to 2018: A Systematic Review and Meta-Analysis. Hepatology 2019. [Epub ahead of print].

8. Matherly SC, Puri P. Mechanisms of simple hepatic steatosis: not so simple after all. Clin Liver Dis 2012;16:505-24.

9. Boutari C, Perakakis N, Mantzoros CS. Association of Adipokines with Development and Progression of Nonalcoholic Fatty Liver Disease. Endocrinol Metab (Seoul) 2018;33:33-43.

10. Wolfs MG, Gruben N, Rensen SS, et al. Determining the association between adipokine expression in multiple tissues and phenotypic features of non-alcoholic fatty liver disease in obesity. Nutr Diabetes 2015;5:e146.

11. Johnson AR, Milner JJ, Makowski L. The inflammation highway: metabolism accelerates inflammatory traffic in obesity. Immunol Rev 2012;249:218-38.

12. Stojsavljevic S, Gomercic Palcic M, Virovic Jukic L, et al. Adipokines and proinflammatory cytokines, the key mediators in the pathogenesis of nonalcoholic fatty liver disease. World J Gastroenterol 2014;20:18070-91.

13. Haukeland JW, Damas JK, Konopski Z, et al. Systemic inflammation in nonalcoholic fatty liver disease is characterized by elevated levels of CCL2. J Hepatol 2006;44:1167-74.

14. Sookoian S, Pirola CJ. Non-alcoholic fatty liver disease is strongly associated with carotid atherosclerosis: a systematic review. J Hepatol 2008;49:600-7.

15. Oni ET, Agatston AS, Blaha MJ, et al. A systematic review: burden and severity of subclinical cardiovascular disease among those with nonalcoholic fatty liver; should we care? Atherosclerosis 2013;230:258-67.

16. Ampuero J, Gallego-Duran R, Romero-Gomez M. Association of NAFLD with subclinical atherosclerosis and coronary-artery disease: meta-analysis. Rev Esp Enferm Dig 2015;107:10-6.

17. Bonci E, Chiesa C, Versacci P, et al. Association of Nonalcoholic Fatty Liver Disease with Subclinical Cardiovascular Changes: A Systematic Review and MetaAnalysis. Biomed Res Int 2015;2015:213737.

18. Madan SA, John F, Pyrsopoulos N, et al. Nonalcoholic fatty liver disease and carotid artery atherosclerosis in children and adults: a meta-analysis. Eur J Gastroenterol 
Hepatol 2015;27:1237-48.

19. Wu S, Wu F, Ding Y, et al. Association of non-alcoholic fatty liver disease with major adverse cardiovascular events: A systematic review and meta-analysis. Sci Rep 2016;6:33386.

20. Zhou YY, Zhou XD, Wu SJ, et al. Nonalcoholic fatty liver disease contributes to subclinical atherosclerosis: A systematic review and meta-analysis. Hepatol Commun 2018;2:376-92.

21. Kapuria D, Takyar VK, Etzion O, et al. Association of Hepatic Steatosis With Subclinical Atherosclerosis: Systematic Review and Meta-Analysis. Hepatol Commun 2018;2:873-83.

22. Vilar CP, Cotrim HP, Florentino GS, et al. Association between nonalcoholic fatty liver disease and coronary artery disease. Rev Assoc Med Bras (1992) 2013;59:290-7.

23. Targher G, Bertolini L, Poli F, et al. Nonalcoholic fatty liver disease and risk of future cardiovascular events among type 2 diabetic patients. Diabetes 2005;54:3541-6.

24. Hamaguchi M, Kojima T, Takeda N, et al. Nonalcoholic fatty liver disease is a novel predictor of cardiovascular disease. World J Gastroenterol 2007;13:1579-84.

25. Targher G, Bertolini L, Rodella S, et al. Nonalcoholic fatty liver disease is independently associated with an increased incidence of cardiovascular events in type 2 diabetic patients. Diabetes Care 2007;30:2119-21.

26. Boddi M, Tarquini R, Chiostri M, et al. Nonalcoholic fatty liver in nondiabetic patients with acute coronary syndromes. Eur J Clin Invest 2013;43:429-38.

27. Dunn MA, Behari J, Rogal SS, et al. Hepatic steatosis in diabetic patients does not predict adverse liver-related or cardiovascular outcomes. Liver Int 2013;33:1575-82.

28. Targher G, Byrne CD, Lonardo A, et al. Non-alcoholic fatty liver disease and risk of incident cardiovascular disease: A meta-analysis. J Hepatol 2016;65:589-600.

29. Zhou YY, Zhou XD, Wu SJ, et al. Synergistic increase in cardiovascular risk in diabetes mellitus with nonalcoholic fatty liver disease: a meta-analysis. Eur J Gastroenterol Hepatol 2018;30:631-6.

30. Kovalic AJ, Satapathy SK. The Role of Nonalcoholic Fatty Liver Disease on Cardiovascular Manifestations and Outcomes. Clin Liver Dis 2018;22:141-74.

31. Kotronen A, Joutsi-Korhonen L, Sevastianova K, et al. Increased coagulation factor VIII, IX, XI and XII activities in non-alcoholic fatty liver disease. Liver Int 2011;31:176-83.

32. Yu KJ, Zhang MJ, Li Y, et al. Increased whole blood viscosity associated with arterial stiffness in patients with non-alcoholic fatty liver disease. J Gastroenterol Hepatol 2014;29:540-4.

33. Di Minno MN, Tufano A, Rusolillo A, et al. High prevalence of nonalcoholic fatty liver in patients with idiopathic venous thromboembolism. World J Gastroenterol 2010;16:6119-22.

34. Tripodi A, Fracanzani AL, Primignani M, et al. Procoagulant imbalance in patients with non-alcoholic fatty liver disease. J Hepatol 2014;61:148-54.

35. Musso G, Gambino R, Tabibian JH, et al. Association of non-alcoholic fatty liver disease with chronic kidney disease: a systematic review and meta-analysis. PLoS Med 2014;11:e1001680.

36. Mantovani A, Zaza G, Byrne CD, et al. Nonalcoholic fatty liver disease increases risk of incident chronic kidney disease: A systematic review and meta-analysis. Metabolism 2018;79:64-76.

37. Baranova A, Tran TP, Birerdinc A, et al. Systematic review: association of polycystic ovary syndrome with metabolic syndrome and non-alcoholic fatty liver disease. Aliment Pharmacol Ther 2011;33:801-14.

38. Rocha ALL, Faria LC, Guimaraes TCM, et al. Nonalcoholic fatty liver disease in women with polycystic ovary syndrome: systematic review and meta-analysis. J Endocrinol Invest 2017;40:1279-88.

39. Wu J, Yao XY, Shi RX, et al. A potential link between polycystic ovary syndrome and non-alcoholic fatty liver disease: an update meta-analysis. Reprod Health 2018;15:77.

40. Musso G, Cassader M, Olivetti C, et al. Association of obstructive sleep apnoea with the presence and severity of non-alcoholic fatty liver disease. A systematic review and meta-analysis. Obes Rev 2013;14:417-31.

41. Jin S, Jiang S, Hu A. Association between obstructive sleep apnea and non-alcoholic fatty liver disease: a systematic review and meta-analysis. Sleep Breath 2018;22:841-51.

42. Hallsworth K, Thoma C, Moore S, et al. Non-alcoholic fatty liver disease is associated with higher levels of objectively measured sedentary behaviour and lower levels of physical activity than matched healthy controls. Frontline Gastroenterol 2015;6:44-51.

43. Chastin SF, Mandrichenko O, Helbostadt JL, et al. Associations between objectively-measured sedentary behaviour and physical activity with bone mineral density in adults and older adults, the NHANES study. Bone 2014;64:254-62.

44. Patterson-Buckendahl P. Osteocalcin is a stress-responsive neuropeptide. Endocr Regul 2011;45:99-110. 
45. Yilmaz Y, Kurt R, Eren F, et al. Serum osteocalcin levels in patients with nonalcoholic fatty liver disease: association with ballooning degeneration. Scand J Clin Lab Invest 2011;71:631-6.

46. Dou J, Ma X, Fang Q, et al. Relationship between serum osteocalcin levels and non-alcoholic fatty liver disease in Chinese men. Clin Exp Pharmacol Physiol 2013;40:282-8.

47. Reid P, Holen I. Pathophysiological roles of osteoprotegerin (OPG). Eur J Cell Biol 2009;88:1-17.

48. Yilmaz Y, Yonal O, Kurt R, et al. Serum levels of osteoprotegerin in the spectrum of nonalcoholic fatty liver disease. Scand J Clin Lab Invest 2010;70:541-6.

49. Ishijima M, Rittling SR, Yamashita T, et al. Enhancement of osteoclastic bone resorption and suppression of osteoblastic bone formation in response to reduced mechanical stress do not occur in the absence of osteopontin. J Exp Med 2001;193:399-404.

50. Syn WK, Choi SS, Liaskou E, et al. Osteopontin is induced by hedgehog pathway activation and promotes fibrosis progression in nonalcoholic steatohepatitis. Hepatology 2011;53:106-15.

51. Upala S, Jaruvongvanich V, Wijarnpreecha K, et al. Nonalcoholic fatty liver disease and osteoporosis: a systematic review and meta-analysis. J Bone Miner Metab 2017;35:685-93.

52. Mantovani A, Dauriz M, Gatti D, et al. Systematic review with meta-analysis: non-alcoholic fatty liver disease is associated with a history of osteoporotic fractures but not with low bone mineral density. Aliment Pharmacol Ther 2019;49:375-88.

53. Chung M, Ma J, Patel K, et al. Fructose, high-fructose corn syrup, sucrose, and nonalcoholic fatty liver disease or indexes of liver health: a systematic review and metaanalysis. Am J Clin Nutr 2014;100:833-49.

54. Asgari-Taee F, Zerafati-Shoae N, Dehghani M, et al. Association of sugar sweetened beverages consumption with non-alcoholic fatty liver disease: a systematic review and meta-analysis. Eur J Nutr 2019;58:1759-69.

55. Haghighatdoost F, Salehi-Abargouei A, Surkan PJ, et al. The effects of low carbohydrate diets on liver function tests in nonalcoholic fatty liver disease: A systematic review and meta-analysis of clinical trials. J Res Med Sci 2016;21:53.

56. Tarantino G, Porcu C, Arciello M, et al. Prediction of carotid intima-media thickness in obese patients with low prevalence of comorbidities by serum copper bioavailability. J Gastroenterol Hepatol 2018;33:1511-7.

57. Wongjarupong N, Assavapongpaiboon B, Susantitaphong
P, et al. Non-alcoholic fatty liver disease as a risk factor for cholangiocarcinoma: a systematic review and metaanalysis. BMC Gastroenterol 2017;17:149.

58. Mantovani A, Dauriz M, Byrne CD, et al. Association between nonalcoholic fatty liver disease and colorectal tumours in asymptomatic adults undergoing screening colonoscopy: a systematic review and meta-analysis. Metabolism 2018;87:1-12.

59. Reig M, Gambato M, Man NK, et al. Should Patients With NAFLD/NASH Be Surveyed for HCC? Transplantation 2019;103:39-44.

60. Deng T, Lyon CJ, Bergin S, et al. Obesity, Inflammation, and Cancer. Annu Rev Pathol 2016;11:421-49.

61. Ackerman SE, Blackburn OA, Marchildon F, et al. Insights into the Link Between Obesity and Cancer. Curr Obes Rep 2017;6:195-203.

62. Yoon YS, Kwon AR, Lee YK, et al. Circulating adipokines and risk of obesity related cancers: A systematic review and meta-analysis. Obes Res Clin Pract 2019;13:329-39.

63. Prussick R, Prussick L, Nussbaum D. Nonalcoholic Fatty liver disease and psoriasis: what a dermatologist needs to know. J Clin Aesthet Dermatol 2015;8:43-5.

64. Candia R, Ruiz A, Torres-Robles R, et al. Risk of nonalcoholic fatty liver disease in patients with psoriasis: a systematic review and meta-analysis. J Eur Acad Dermatol Venereol 2015;29:656-62.

65. Scalera A, Di Minno MN, Tarantino G. What does irritable bowel syndrome share with non-alcoholic fatty liver disease? World J Gastroenterol 2013;19:5402-20.

66. Khukhlina OS, Antoniv AA, Mandryk OY, et al. Clinical and pathogenetic features of nonalcoholic steatohepatitis for comorbidity with bronchial asthma on the background of obesity. Wiad Lek 2018;71:376-9.

67. Weinstein AA, Kallman Price J, Stepanova M, et al. Depression in patients with nonalcoholic fatty liver disease and chronic viral hepatitis B and C. Psychosomatics 2011;52:127-32.

68. Rao R. Endotoxemia and gut barrier dysfunction in alcoholic liver disease. Hepatology 2009;50:638-44.

69. Szabo G. Gut-liver axis in alcoholic liver disease. Gastroenterology 2015;148:30-6.

70. Li P, He K, Li J, et al. The role of Kupffer cells in hepatic diseases. Mol Immunol 2017;85:222-9.

71. Gonzalez-Reimers E, Santolaria-Fernandez F, Martin-Gonzalez MC, et al. Alcoholism: a systemic proinflammatory condition. World J Gastroenterol 2014;20:14660-71.

72. Teschke R. Alcoholic Liver Disease: Alcohol Metabolism, 
Cascade of Molecular Mechanisms, Cellular Targets, and Clinical Aspects. Biomedicines 2018. doi: 10.3390/ biomedicines6040106.

73. Moses Elisaf M, Rigas Kalaitzidis M. Metabolic abnormalities in alcoholic patients: focus on acid base and electrolyte disorders. J Alcohol Drug Depend 2015;2:2.

74. Scheiner B, Lindner G, Reiberger T, et al. Acid-base disorders in liver disease. J Hepatol 2017;67:1062-73.

75. Hammoud N, Jimenez-Shahed J. Chronic Neurologic Effects of Alcohol. Clin Liver Dis 2019;23:141-55.

76. Garcia-Valdecasas-Campelo E, Gonzalez-Reimers E, Santolaria-Fernandez F, et al. Brain atrophy in alcoholics: relationship with alcohol intake; liver disease; nutritional status, and inflammation. Alcohol Alcohol 2007;42:533-8.

77. Noble JM, Weimer LH. Neurologic complications of alcoholism. Continuum (Minneap Minn) 2014;20:624-41.

78. Gimsing P, Melgaard B, Andersen K, et al. Vitamin B-12 and folate function in chronic alcoholic men with peripheral neuropathy and encephalopathy. J Nutr 1989;119:416-24.

79. Sureka B, Bansal K, Patidar Y, et al. Neurologic Manifestations of Chronic Liver Disease and Liver Cirrhosis. Curr Probl Diagn Radiol 2015;44:449-61.

80. Roerecke M, Kaczorowski J, Tobe SW, et al. The effect of a reduction in alcohol consumption on blood pressure: a systematic review and meta-analysis. Lancet Public Health 2017;2:e108-20.

81. Samokhvalov AV, Irving HM, Rehm J. Alcohol consumption as a risk factor for atrial fibrillation: a systematic review and meta-analysis. Eur J Cardiovasc Prev Rehabil 2010;17:706-12.

82. Larsson SC, Drca N, Wolk A. Alcohol consumption and risk of atrial fibrillation: a prospective study and doseresponse meta-analysis. J Am Coll Cardiol 2014;64:281-9.

83. Kodama S, Saito K, Tanaka S, et al. Alcohol consumption and risk of atrial fibrillation: a meta-analysis. J Am Coll Cardiol 2011;57:427-36.

84. Gallagher C, Hendriks JML, Elliott AD, et al. Alcohol and incident atrial fibrillation - A systematic review and metaanalysis. Int J Cardiol 2017;246:46-52.

85. Milic S, Lulic D, Stimac D, et al. Cardiac manifestations in alcoholic liver disease. Postgrad Med J 2016;92:235-9.

86. Zardi EM, Abbate A, Zardi DM, et al. Cirrhotic cardiomyopathy. J Am Coll Cardiol 2010;56:539-49.

87. Djousse L, Gaziano JM. Alcohol consumption and heart failure: a systematic review. Curr Atheroscler Rep 2008;10:117-20.

88. Rehm J, Hasan OSM, Imtiaz S, et al. Quantifying the contribution of alcohol to cardiomyopathy: A systematic review. Alcohol 2017;61:9-15.

89. Krumpe PE, Cummiskey JM, Lillington GA. Alcohol and the respiratory tract. Med Clin North Am 1984;68:201-19.

90. Kim AC, Epstein ME, Gautam-Goyal P, et al. Infections in alcoholic liver disease. Clin Liver Dis 2012;16:783-803.

91. Rebetz J, Semple JW, Kapur R. The Pathogenic Involvement of Neutrophils in Acute Respiratory Distress Syndrome and Transfusion-Related Acute Lung Injury. Transfus Med Hemother 2018;45:290-8.

92. Hewitt SM, Winter RJ. Rhabdomyolysis following acute alcohol intoxication. J Accid Emerg Med 1995;12:143-4.

93. Htet Z. Alcohol-induced Rhabdomyolysis: A Disease With Potential Pitfalls. Acute Med 2018;17:226-8.

94. Lang CH, Pruznak AM, Deshpande N, et al. Alcohol intoxication impairs phosphorylation of S6K1 and S6 in skeletal muscle independently of ethanol metabolism. Alcohol Clin Exp Res 2004;28:1758-67.

95. Thapaliya S, Runkana A, McMullen MR, et al. Alcoholinduced autophagy contributes to loss in skeletal muscle mass. Autophagy 2014;10:677-90.

96. Simon L, Jolley SE, Molina PE. Alcoholic Myopathy: Pathophysiologic Mechanisms and Clinical Implications. Alcohol Res 2017;38:207-17.

97. Mendenhall CL, Anderson S, Weesner RE, et al. Proteincalorie malnutrition associated with alcoholic hepatitis. Veterans Administration Cooperative Study Group on Alcoholic Hepatitis. Am J Med 1984;76:211-22.

98. Lieber CS. Relationships between nutrition, alcohol use, and liver disease. Alcohol Res Health 2003;27:220-31.

99. Testino G. Alcoholic diseases in hepato-gastroenterology: a point of view. Hepatogastroenterology 2008;55:371-7.

100. Styskel B, Natarajan Y, Kanwal F. Nutrition in Alcoholic Liver Disease: An Update. Clin Liver Dis 2019;23:99-114.

101. Kalaitzakis E, Bosaeus I, Ohman L, et al. Altered postprandial glucose, insulin, leptin, and ghrelin in liver cirrhosis: correlations with energy intake and resting energy expenditure. Am J Clin Nutr 2007;85:808-15.

102.Peng S, Plank LD, McCall JL, et al. Body composition, muscle function, and energy expenditure in patients with liver cirrhosis: a comprehensive study. Am J Clin Nutr 2007;85:1257-66.

103. Dasarathy S. Cause and management of muscle wasting in chronic liver disease. Curr Opin Gastroenterol 2016;32:159-65.

104. Dasarathy J, McCullough AJ, Dasarathy S. Sarcopenia in Alcoholic Liver Disease: Clinical and Molecular Advances. Alcohol Clin Exp Res 2017;41:1419-31. 
105.Skalny AV, Skalnaya MG, Grabeklis AR, et al. Zinc deficiency as a mediator of toxic effects of alcohol abuse. Eur J Nutr 2018;57:2313-22.

106.Pezzilli R, Caputo F, Testino G, et al. Alcohol-related chronic exocrine pancreatic insufficiency: diagnosis and therapeutic management. A proposal for treatment by the Italian Association for the Study of the Pancreas (AISP) and the Italian Society of Alcohology (SIA). Minerva Med 2019;110:425-38.

107.Struyvenberg MR, Martin CR, Freedman SD. Practical guide to exocrine pancreatic insufficiency - Breaking the myths. BMC Med 2017;15:29.

108. Duggan SN. Negotiating the complexities of exocrine and endocrine dysfunction in chronic pancreatitis. Proc Nutr Soc 2017;76:484-94.

109. Wakolbinger R, Muschitz C, Scheriau G, et al. Bone microarchitecture and bone turnover in hepatic cirrhosis. Osteoporos Int 2019;30:1195-204.

110. Kizilgul M, Ozcelik O, Delibasi T. Bone health and vitamin $\mathrm{D}$ status in alcoholic liver disease. Indian $\mathrm{J}$ Gastroenterol 2016;35:253-9.

111. Stigliano S, Waldthaler A, Martinez-Moneo E, et al. Vitamins D and K as Factors Associated with Osteopathy in Chronic Pancreatitis: A Prospective Multicentre Study (P-BONE Study). Clin Transl Gastroenterol 2018;9:197.

112.Gonzalez-Reimers E, Quintero-Platt G, RodriguezRodriguez E, et al. Bone changes in alcoholic liver disease. World J Hepatol 2015;7:1258-64.

113. Giuliani N, Girasole G, Vescovi PP, et al. Ethanol and acetaldehyde inhibit the formation of early osteoblast progenitors in murine and human bone marrow cultures.

doi: $10.21037 / \operatorname{tgh} .2019 .08 .09$

Cite this article as: Kovalic AJ, Cholankeril G, Satapathy SK. Nonalcoholic fatty liver disease and alcoholic liver disease: metabolic diseases with systemic manifestations. Transl Gastroenterol Hepatol 2019;4:65.
Alcohol Clin Exp Res 1999;23:381-5.

114.Fabrega E, Orive A, Garcia-Suarez C, et al.

Osteoprotegerin and RANKL in alcoholic liver cirrhosis. Liver Int 2005;25:305-10.

115. Bang CS, Shin IS, Lee SW, et al. Osteoporosis and bone fractures in alcoholic liver disease: a meta-analysis. World J Gastroenterol 2015;21:4038-47.

116. Peris P, Pares A, Guanabens N, et al. Bone mass improves in alcoholics after 2 years of abstinence. J Bone Miner Res 1994;9:1607-12.

117. Roerecke M, Rehm J. Cause-specific mortality risk in alcohol use disorder treatment patients: a systematic review and meta-analysis. Int J Epidemiol 2014;43:906-19.

118.IARC Working Group on the Evaluation of Carcinogenic Risks to Humans. Alcohol consumption and ethyl carbamate. IARC Monogr Eval Carcinog Risks Hum 2010;96:3-1383.

119. Boyle P, Boffetta P. Alcohol consumption and breast cancer risk. Breast Cancer Res 2009;11 Suppl 3:S3.

120.Wang Y'T, Gou YW, Jin WW, et al. Association between alcohol intake and the risk of pancreatic cancer: a doseresponse meta-analysis of cohort studies. BMC Cancer 2016;16:212.

121. Merlo C, Bohl L, Carda C, et al. Parotid sialosis: morphometrical analysis of the glandular parenchyme and stroma among diabetic and alcoholic patients. J Oral Pathol Med 2010;39:10-5.

122.Lurati AR. Dupuytren's Contracture. Workplace Health Saf 2017;65:96-9.

123. Smith KE, Fenske NA. Cutaneous manifestations of alcohol abuse. J Am Acad Dermatol 2000;43:1-16; quiz 16-8. 